\title{
Two-Photon Excitation of a Plasmonic Nanoswitch Monitored by Single Molecule Fluorescence Microscopy
}

\author{
Stefania Impellizzeri, ${ }^{[a]}$ Sabrina Simoncelli, ${ }^{[a, b]}$ Gregory K. Hodgson, ${ }^{[a]}$ Anabel E. Lanterna, ${ }^{[a]}$ Christopher \\ D. McTiernan, ${ }^{\left[{ }^{[a]}\right.}$ Françisco M. Raymo, ${ }^{[\mathrm{c}]}$ Pedro F. Aramendia, ${ }^{[\mathrm{d}]}$ and Juan. C. Scaiano), ${ }^{\text {[a] }}$
}

\begin{abstract}
Visible light excitation of the surface plasmon band of silver nanoplates can effectively localize and concentrate the incident electromagnetic field enhancing the photochemical performance of organic molecules. Herein, the first single-molecule study of the plasmon-assisted isomerization of a photochromefluorophore dyad, designed to switch between a nonfluorescent and a fluorescent state in response to the photochromic transformation, is reported. The photochemistry of the switchable assembly, consisting of a photochromic benzooxazine chemically conjugated to a coumarin moiety, is examined in real time with Total Internal Reflection Fluorescence Microscopy in the presence of silver nanoplates excited with a $633 \mathrm{~nm}$ laser. The metallic nanostructures significantly enhance the visible light-induced performance of the photoconversion, which normally requires ultraviolet excitation. The resulting ring-open isomer is strongly fluorescent and can also be excited at $633 \mathrm{~nm}$. These stochastic emission events are used to monitor photochromic activation and show quadratic dependence on incident power. The utilization of a single laser wavelength for both photochromic activation and excitation effectively mimics a pseudotwo-colour system.
\end{abstract}

\section{Introduction}

The utilization of surface plasmon resonance (SPR) of noble metal nanoparticles (MNP) to manipulate light in the subwavelength regime with spatial and temporal control is of great interest due to potential applications in nanoscaled lithography, data storage and microscopy. ${ }^{[1]}$ The SPR band is generated by the collective oscillation of free electrons upon interaction of the

[a] Dr. S. Impellizzeri, G. K. Hodgson, Dr. A. E. Lanterna, C. D. McTiernan, Prof. J. C. Scaiano

Department of Chemistry and Biomolecular Sciences, University of Ottawa

10 Marie Curie, Ottawa, Ontario K1N 6N5, Canada

E-mail: scaiano@photo.chem.uottawa.ca

[b] Dr. S. Simoncelli

Center for NanoScience (CeNS), Faculty of Physics, Ludwig Maximilians Universität München, Amalienstraße 54, 80799 Munich, Germany

[c] Prof. F. M. Raymo

Laboratory for Molecular Photonics

Department of Chemistry, University of Miami

1301 Memorial Drive, Coral Gables, FL 33146-0431 (USA)

[d] Prof. P. A. Aramendia

Centro de Investigaciones en Bionanociencias (CIBION) CONICET Godoy Cruz 2390

Departamento de Química Inorgánica, Analítica y Química Física, FCEN, UBA, Pabellón 2, Ciudad Universitaria

Buenos Aires, Argentina

Supporting information for this article is given via a link at the end of the document.
MNP with visible light. Conveniently, the shape and the spectral profile of the SPR band can be regulated by the changing size, morphology and surface coverage of the MNP. ${ }^{[2]}$ When excited in the plasmon region, silver nanoparticles dramatically intensify the electromagnetic field and thus the photon flux experienced by molecules in the direct vicinity of the particles. ${ }^{[2 b, 3]}$ This consequently amplifies the inherent spectroscopic and photochemical behavior of molecules in close proximity to the metal surface, improving the efficiency and the performance of photochemical reactions in the visible and near-infrared wavelength regime. Interestingly, photochromic transformations have been successfully exploited for tailoring, probing and monitoring SPR effects with convenient spectroscopic measurements. ${ }^{[4]}$ Photochromic compounds switch reversibly between two isomeric states with distinct absorption spectra under optical control. ${ }^{[5]}$ While photochromic transformations normally involve single-photon photoexcitation of the organic chromophore, the nanoparticle-mediated plasmonic enhancement of the electromagnetic field allows for the stimulation of multi-photon photochromic processes even at moderate illumination intensities that would otherwise be inadequate for two-photon excitation. ${ }^{[6]}$ In this context, nanoparticles can act as antennae, delivering excitation energy to molecules in close proximity to their surfaces. A number of examples have been reported, including a successful twophoton ring opening of diarylethenes. ${ }^{[4 c, 4 d]}$ Since however, these systems can only be monitored by absorption techniques, they are not amenable for single-molecule fluorescence microscopy studies.

Photochromic transformations can instead be designed to activate fluorescence under optical control, owing to the chemical engineering of the conjugation between a photochromic unit and an organic fluorophore. ${ }^{[7]}$ As a consequence of the pronounced structural and electronic modifications associated with a photochromic transformation, the light-induced interconversion of the photochromic unit can be exploited to switch on the emission of the complementary fluorophore. The activation of fluorescence upon photochromic conversion offers the opportunity to apply single-molecule techniques, where the photogenerated emission translates into a detectable signal for stochastic events at the single-molecule level. ${ }^{[8]}$ For this study, we have selected the photochromic system shown in Figure 1. Upon ultraviolet illumination in acetonitrile, the $2 \mathrm{H}, 4 \mathrm{H}-[1,3]$ benzooxazine ring in $1 \mathrm{a}$ opens to generate the zwitterionic isomer $\mathbf{1 b}$ on a subnanosecond time scale, where $\mathbf{1 b}$ spontaneously reverts to the original isomer 1a. ${ }^{[9][10]}$ The structural transformation of the photochrome brings the coumarin moiety into conjugation with the cationic fragment of the light-generated isomer and bathochromically shifts its absorption band by ca. $160 \mathrm{~nm}$. As a result, the selective excitation of the newly generated absorption band centered at 
$580 \mathrm{~nm}$ allows the detection of the fluorescence of this species at $685 \mathrm{~nm}$, which is readily observable following the photochromic transformation. The generation of an emissive isomer allows for monitoring of the photochromic conversion in real time, using Total Internal Reflection Fluorescence Microscopy (TIRFM). The conversion of $\mathbf{1 a}$ into $\mathbf{1 b}$ normally requires excitation at wavelengths shorter than $450 \mathrm{~nm}$, and is typically achieved using ultraviolet light. Herein, we used silver nanoplates (AgNP) in an attempt to establish whether the transformation shown in Figure 1 could instead be stimulated by excitation at $633 \mathrm{~nm}$. Whereas compound $1 \mathrm{a}$ is transparent at this wavelength, AgNP show strong plasmon absorption (Figure S1, Supporting Information). Furthermore, the transient absorption spectrum of $\mathbf{1 b}$ (Figure S1) shows that $633 \mathrm{~nm}$ is also a suitable excitation wavelength to observe fluorescence. This design allows both activation (the transformation of 1a into 1b) and excitation (of $\mathbf{1 b}$ ) to be conveniently achieved by the utilization of a single laser, simplifying the experimental setup and minimizing the associated cost. Our efforts were thus directed at the possibility to achieve fluorescence activation via the plasmon-mediated photochromic transformation of $\mathbf{1 a}$, as a consequence of the visible excitation of the SPR band of silver nanoplates (rather than the organic chromophore), and to exploit this process to detect emissive molecules. Results show that this transformation is indeed possible, and suggest a non-linear process attributed to the plasmon-mediated enhancement of the performance of the photochromic transformation facilitated by the interaction of $1 \mathrm{a}$ with the strong plasmonic field.
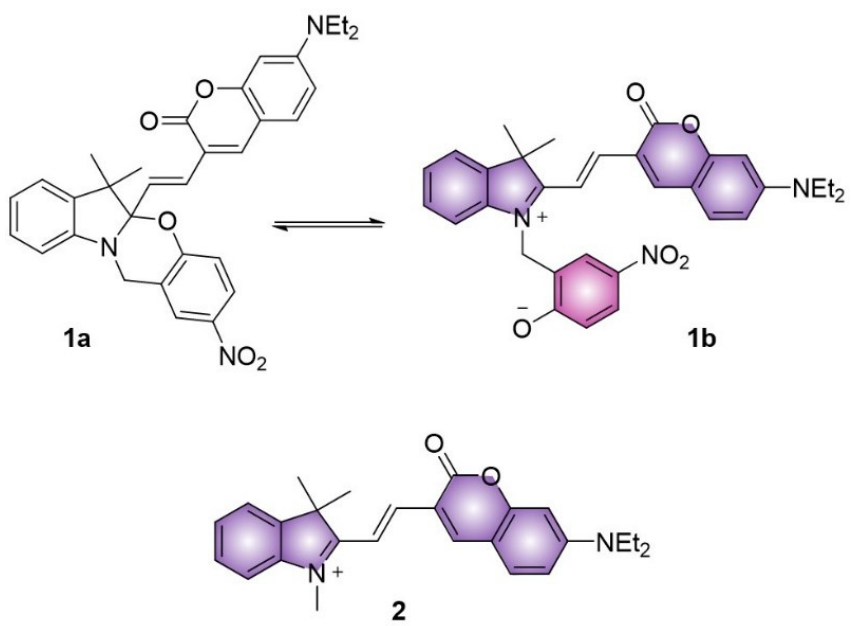

Figure 1. Photochromic transformation of $\mathbf{1 a}$ into $\mathbf{1 b}$ and structure of the model cationic compound 2 .

\section{Results and Discussion}

TIRFM samples consisted of a thin polymer layer of poly(methyl methacrylate) (PMMA) doped with 1a and deposited over a monolayer of triangular shaped AgNPs coated on a glass coverslip. Scanning Electron Microscopy (SEM) of the synthesized AgNP (Figure S2, Supporting Information) reveal triangular shaped nanocrystals with size distribution of $90 \pm 9 \mathrm{~nm}$. Relative to their spherical counterparts, triangular silver plates are known to generate stronger plasmonic fields, which are localized at the vertices of the prisms. ${ }^{[11]}$ The formation of clusters of closely spaced nanoparticles can further enhance performance by constraining plasmon effects to the nanoscaled gaps between the vertices. ${ }^{[12]}$ The AgNP monolayer was prepared by using APTES as a coupling agent. AFM images of the substrates show the presence of large AgNP agglomerates as well as complete surface coverage of the substrate (Figure S3, Supporting Information). A solution of 1 a in poly(methyl methacrylate) was then spin coated atop the functionalized slides in order to obtain polymer films approximately $100 \mathrm{~nm}$ thick. The construction and the preparation of the sample ensures that molecules dispersed atop the AgNP monolayer will be in close proximity to the enhanced field produced by excitation of the localized surface plasmon of AgNP, with the gaps between the densely packed nanostructures filled by polymer. ${ }^{[13]}$ With full coverage of the slide with AgNP and a polymer film $100 \mathrm{~nm}$ thick, the enhanced field is expected to cover roughly $20 \%$ of the sample. ${ }^{[14]}$ This is sufficient to ensure that most molecules of $1 \mathrm{a}$ will be sufficiently close to AgNP and within the field enhancement region. A fraction of the small population of $\mathbf{1 b}$ in thermal equilibrium with $\mathbf{1 a}$ will experience field enhancement. TIRFM imaging and analysis of a PMMA solution of 1a spin coated atop a AgNP monolayer after $633 \mathrm{~nm}$ excitation at full laser power density $\left(83 \mathrm{~W} / \mathrm{cm}^{2}\right)$ reveal a significant increase in the mean fluorescence intensity as well as in the number of detected events with respect to $1 \mathrm{a}$ imaged in the absence of the AgNP (see Supporting Video 1). For an immediate visual comparison, Figure 2 displays the contrast between two regions of a coverslip, where only one of them is functionalized with AgNP. Small populations of fluorescent molecules are evident even in the absence of AgNP, as a consequence of spontaneous thermal activation due to equilibrium between the closed (1a) and the open form (1b). The emission spectrum of such bursting events confirmed that they indeed correspond to emission from $\mathbf{1 b}$, as shown in Figure 2c.

AgNP monolayers on a glass coverslip are highly scattering under the selected experimental conditions. The background scattering from AgNP, measured by imaging PMMA spin coated atop the nanostructures, was systematically analysed at different excitation powers in order to evaluate possible interference in identifying actual events of interest. The temporal intensity profiles for representative background trajectories are shown in Figure S4 (Supporting Information). Although the average base intensity of each scattering trajectory may vary according to the non-uniform distribution of the NP on the glass surface, on the time scale of the experiment (100 s, Figure S4, Supporting Information) it remains approximately constant for a given laser power, and in no cases were bursting signals or sharp jumps in intensity observed. Indeed, analysis of residuals of the scattering profiles (Figure S5, Supporting Information) show that the variance is normally distributed, which confirms the normality of the background signals over time. In contrast, analysis of the variance of the residuals for intensity trajectories extracted from 

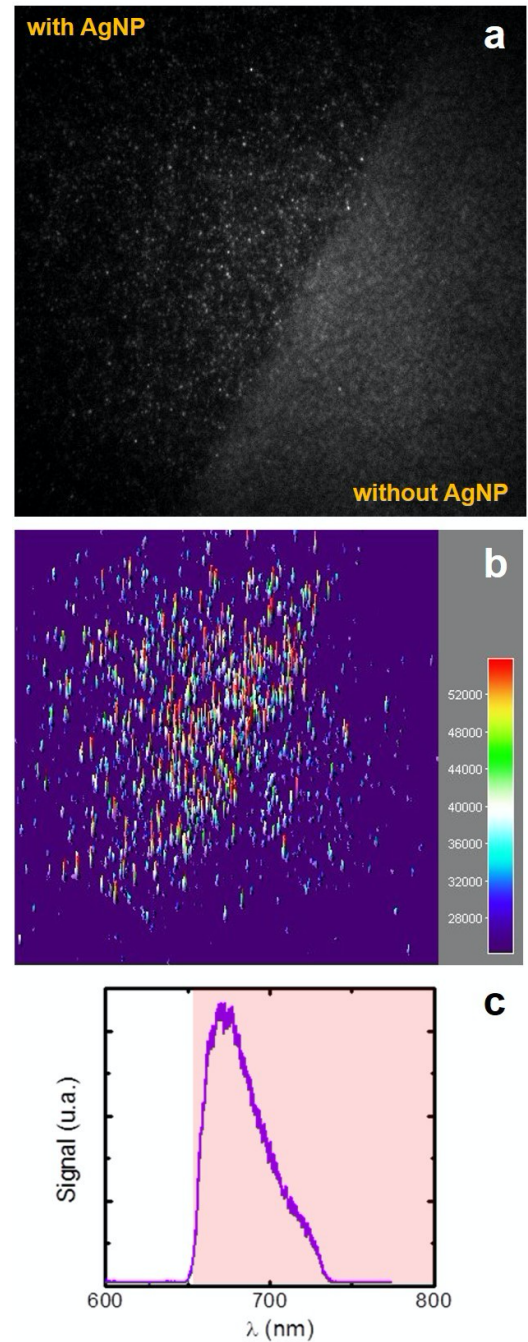

C

Figure 2. Single frame $(\boldsymbol{a})$ and $3 \mathrm{D}$ representation (b) from TIRFM image sequences (100 frames/image sequence, integration time per frame $=999$ $\mathrm{ms}$ ) of a PMMA film doped with 1 a $\left(10^{-6} \mathrm{~mol} / \mathrm{kg}\right.$ polymer $)$ recorded upon illumination with a CW laser operating at $633 \mathrm{~nm}$ over a cover glass where roughly the left half is functionalized with AgNP. Emission spectrum (c) of the fluorescent bursts measured with an Andor spectrograph

TIRFM image sequences corresponding to PMMA films containing 1a $\left(10^{-6} \mathrm{~mol} / \mathrm{kg}\right.$ pol) spin coated atop AgNP shows numerous instances of substantial drift from linear behavior. This phenomenon is indicative of the presence of bursting events in an intensity trajectory. Since this method considers only the absolute drift in the variance, real bursting events are identified independent of their intensities, and can thus be detected even in cases where scattering from dense clusters of nanoparticles (NP) precludes the intensity-based identification of the emission of a few single molecules directly atop the NP. Being completely independent of relative profile intensities, this method relies upon the absolute drift in signal variance in order to identify trajectories containing valid bursting events, which are immediately detected, while scattering profiles are excluded from further analysis. To better illustrate the validity of the analysis, an illustrative example is represented in Figure S6 (for

scattering) and Figure S7 (for a bursting event) of the Supporting Information. Indeed, the protocol described allows for rapid, streamlined identification of ROls that contain potential bursting events by permitting a preliminary screening of thousands of trajectories in a fraction of the time that would be required for manual burst identification, and with significantly greater accuracy. However, manual visual examination of the individual traces making up the pool of trajectories remaining after the screening process was still necessary in order to identify trajectories containing multiple bursting events (Figure S8a) and to confirm that each and every bursting event contained within these trajectories was valid. That is, trajectories affected by camera artefacts or the presence of dust particles on the optics or sample surface could potentially pass the automated screening test if any of these effects resulted in a substantial drift to the variance of the residual. Such trajectories were excluded from further analysis, and any apparent bursting events in which the entire duration of the burst was not observed from start to finish were also discarded (Figure S8b and S8c, Supporting Information). Once the procedure described above had been applied, the actual bursting events were manually counted. The number of counted events as a function of the power of the exciting laser for compounds $\mathbf{1 a}$ and $\mathbf{2}$ in the presence of AgNP, as well as for compound 1a in the absence of the MNP, is illustrated in Figure $\mathbf{3}$ and Table S1 (Supporting Information). Control experiments (compound 1a in the absence of AgNP and compound 2 in the presence of AgNP) were performed by imaging samples at $4,6,20$ and $48 \%$ of full laser power, while for the investigation of compound $1 \mathrm{a}$ in the presence of AgNP intermediate excitation powers were also included.

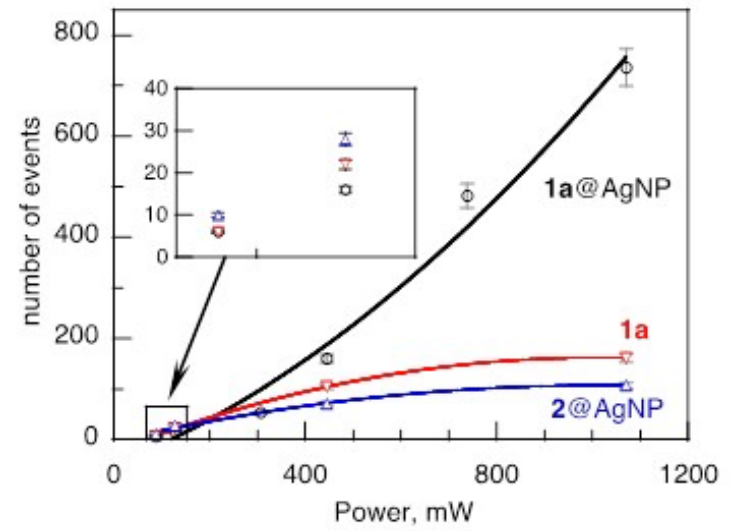

Figure 3. Number of counted events as a function of the power of the exciting laser for PMMA films doped with compounds $\mathbf{1 a}$ or $\mathbf{2}$ in the presence of AgNP and compound 1a in the absence of AgNP, recorded upon illumination with a CW laser operating at $633 \mathrm{~nm}$ (100 frames/image sequence, integration time per frame $=999 \mathrm{~ms}$ ). The errors are estimated as \pm 2 events or $5 \%$, whichever is larger. The quadratic fits are simply to help visualize the data and is only meaningful in the case of 1a@AgNP. $100 \%$ of the laser power corresponds to $2231 \mathrm{~mW}$. 
From the results illustrated in Figure 3 and Table S1 it is clear that the number of detected fluorescent bursts attributed to $\mathbf{1 b}$ in the presence of AgNP is significantly higher than the number observed for the compound imaged in the absence of AgNP. In principle, this remarkable behavior could be the result of: 1) nanoparticle-induced enhancement of the emission intensity of the small population of $\mathbf{1 b}$ present in equilibrium with $\mathbf{1 a}$ (i.e., events are detected more easily due to enhancement of the fluorescence); 2) thermally activated transformation of $\mathbf{1 a}$ to $\mathbf{1 b}$ via plasmonic heating, or 3 ) the ability of the AgNP to promote the formation of $\mathbf{1 b}$ upon excitation of the SP band through a two-photon process.

In order to rule out the first hypothesis, we conducted control experiments by imaging a solution of compound 2 in PMMA spin coated atop AgNP. Compounds $\mathbf{2}$ and $\mathbf{1 b}$ share the same chromophore and thus the analysis of $\mathbf{2}$ atop AgNP provides reliable evidence of the expected enhancement for $\mathbf{1 b}$ atop AgNP at the same laser power (Figure S9, Supporting Information). However, the dependence of the number of events on the power of the exciting laser differs significantly from a sample comprising 1a atop AgNP in PMMA. Double logarithmic plots (Figure 4) show that the dependence of number of bursting events on the excitation power for films of $1 \mathrm{a}$ in the presence of AgNP has a slope $m$ of 2.0 (Figure 4a). In contrast, compound 2 spin coated atop AgNP (Figure 4c) shows a dependence closer to a slope $m$ of 1 , albeit with some curvature in the plot, similar also to the film doped with $1 \mathbf{a}$ in the absence of the nanostructures (Figure 4b). This behaviour indicates that only in the case of $1 \mathrm{a} @ A g N P$ there is a clear non-linear dependence corresponding to a two-photon process, also justifying the use of a parabola for this specific system in Figure 3. These data demonstrate that the nanoplates promote the transformation of a significant population of $\mathbf{1 a}$ into $\mathbf{1 b}$ upon excitation of the SP band at $633 \mathrm{~nm}$. The value of the slope together with the lack of absorbance of 1a at $633 \mathrm{~nm}$ (Figure S1, Supporting Information) show that two-photon excitation at $633 \mathrm{~nm}$, promoted by the stimulation of the surface plasmon band of AgNP at the same wavelength, is responsible for improving the performance of the photochromic transformation of $\mathbf{1 a}$ into $\mathbf{1 b}$.

Our second hypothesis above involves the possibility of thermal activation of $1 \mathrm{a}$. The area in close proximity to the surface of MNP can reach high temperatures upon excitation of the plasmon band. ${ }^{[15]}$ In order to evaluate whether the ring-opening reaction (1a $\rightarrow$ 1b) could be due to thermal stimulation, we prepared thin PMMA films of 1 a deposited over coverslips functionalized with spherical gold nanoparticles (AuNP, $80 \mathrm{~nm}$ in diameter) and recorded TIRFM image sequences upon excitation of the AuNP plasmon band at $543 \mathrm{~nm}$. Compound 1b also absorbs at $543 \mathrm{~nm}$ (Figure S1, Supporting Information) and thus can also be excited at this wavelength in order to detect its fluorescence. Due to the impracticality of physically heating the microscope objective at high temperatures without seriously damaging the equipment, the AuNP based system was chosen as a reliable model to test whether the isomerization was
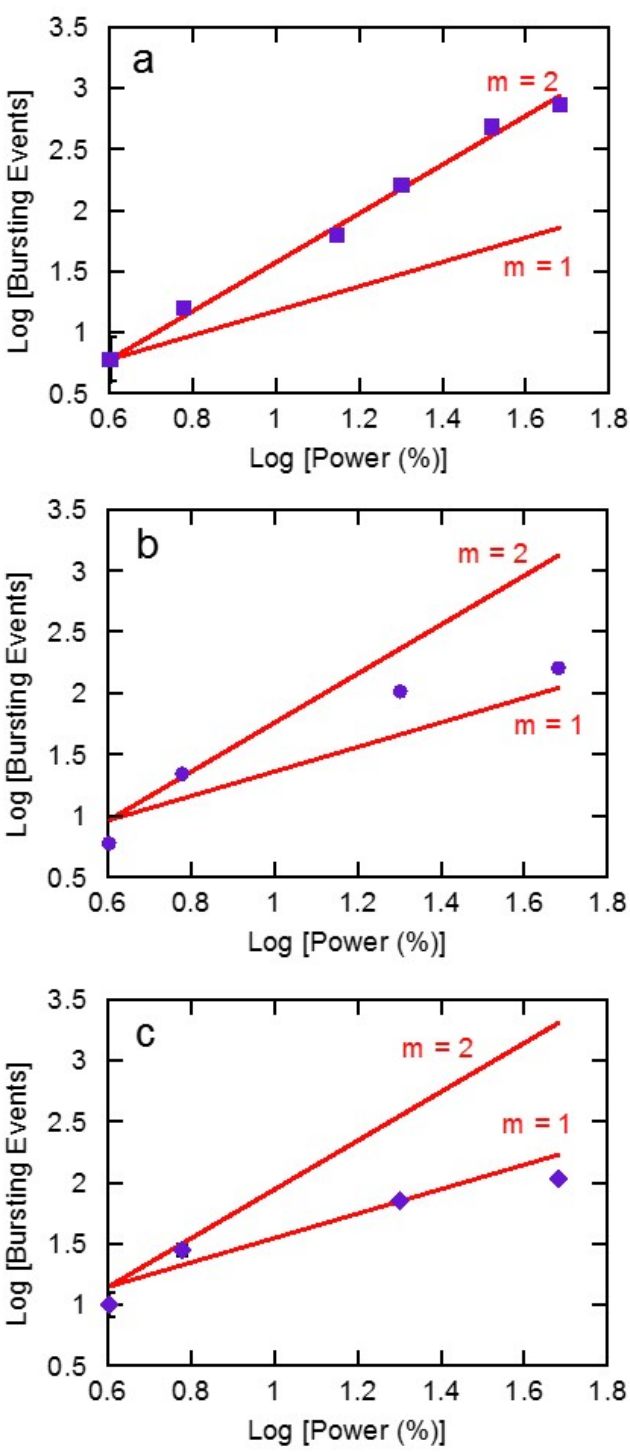

Figure 4. Logarithmic plots of the number of detected bursting events (100 frames/image sequence, integration time per frame $=999 \mathrm{~ms}$ ) for PMMA films doped with compounds $\mathbf{1 a}(\mathbf{a})$ in the presence of AgNP, compound 1a in the absence of AgNP (b) and 2 in the presence of AgNP (c), recorded upon illumination with a $\mathrm{CW}$ laser operating at $633 \mathrm{~nm}$. The errors are estimated as \pm 2 events or $5 \%$, whichever is larger. For $x$-axis values larger than 1 , the errors are within the dimensions of the point drawn; some of these are more readily visualized in Figure 3.

photochemically or thermally driven. Indeed, the local field enhancement produced by spherical AuNP is significantly smaller than for AgNP with triangular geometry ${ }^{[11-12,16]}$ and therefore a plasmon assisted two-photon isomerization process would be unlikely. However, light-to-heat conversion remains highly effective. ${ }^{[17]}$ It should be noted that the parameters for this study require careful selection of experimental conditions. The use of a different material (gold vs. silver), a different laser excitation wavelength, and different plasmonic properties are all important considerations in the quantification of the heating by gold and silver nanostructures. However, most of these 
parameters are readily available or easy to estimate (see Supporting Information). In this case we have concluded that 80 $\mathrm{nm}$ AuNP excited at $543 \mathrm{~nm}$ provide a reasonable comparison through which to evaluate the possible contribution of plasmonic heating to the $\mathbf{1 a} \rightarrow \mathbf{1 b}$ interconversion. According to our calculations, the heat generated via excitation of spherical AuNP at $543 \mathrm{~nm}$ using $100 \%$ of the laser power is comparable to that generated by excitation of AgNP at $633 \mathrm{~nm}$ at $15.7 \%$ of excitation capacity (see Supporting Information). Using the power settings available at $633 \mathrm{~nm}$, we detected 63 events for polymer films doped with 1a and spin coated atop AgNP at $14 \%$ of the total excitation power at $\lambda_{\mathrm{Ex}}=633 \mathrm{~nm}$ and 160 events at $20 \%$ power (Table S1). If the experimental observations in Figure $4 a$ resulted from significant thermal activation, then imaging of samples of $\mathbf{1 a}$ in PMMA spin coated atop AuNP should provide a number between 63-160 events. However, following the same protocol for counting bursting events described above, we detected only 9 events attributable to $\mathbf{1 b}$ atop AuNP. Therefore, the heat generated upon excitation of the $\mathrm{SP}$ of AgNP is insufficient for promoting the conversion of $1 \mathrm{a}$ into 1b. Having eliminated nanoparticle-induced enhancement of emission and thermal activation as possible causes for the light induced $1 \mathrm{a} \rightarrow \mathbf{1 b}$ conversion, the only reasonable explanation remains that AgNP are able to promote the formation of $\mathbf{1 b}$ through a two-photon process mediated by laser excitation of the surface plasmon band of AgNP in the visible region where 1a has no absorption.

\section{Conclusions}

We have demonstrated that visible light excitation of the surface plasmon band of AgNP improves the performance of an activatable photochromic dyad that is transparent in the visible region. In the presence of AgNP, a photoinduced photochromic isomerization that normally requires ultraviolet irradiation $(1 \mathrm{a} \rightarrow$ 1b) can be performed with moderate excitation powers using a simple experimental setup. Results indicate that AgNP effectively promote two-photon excitation, and consequently enhance the fluorescence activation of the switchable probe. Our approach demonstrates that even with photochromic systems that do not display any photochemical activity under visible light illumination, the use of plasmon-enhanced fields can improve performance and allow real time monitoring of the dynamic process using single-molecule fluorescence microscopy.

\section{Experimental Section}

\section{Materials}

Silver nanoplates (AgNP) were prepared according to literature protocols. [4] Specifically, silver seeds were obtained by 5 min UVA illumination of an aerated solution of $0.2 \mathrm{mM}$ Irgacure 2959, $0.2 \mathrm{mM} \mathrm{AgNO}_{3}$ and $1 \mathrm{mM}$ trisodium citrate in a Luzchem photoreactor. The growth from nanoparticles to nanoplates was performed by illuminating an oxygenated solution of the seeds with $590 \mathrm{~nm}$ air-cooled LEDs for $24 \mathrm{~h}$. SEM images were acquired with a JEOL JSM-7500F field emission scanning electron microscope. Gold nanoparticles (AuNP) were prepared with a growth seed method according to literature protocol. [31] Compounds $\mathbf{1 a}$ and $\mathbf{2}$ were synthesized following literature procedures. [16]

\section{Methods}

Solvents were purified with a LC Technology Solutions Inc. SPBT-1 Bench Top Solvent Purification System. Chemicals were purchased from Sigma-Aldrich or Fisher Scientific. All the reactions were monitored by thin-layer chromatography, using aluminum sheets coated with silica $(60$, F254). NMR spectra were recorded at room temperature with a Bruker Avance 300. Mass spectral analysis was performed with a $6890 \mathrm{~N}$ Network GC System equipped with a 5973 Mass Selective Detector from Agilent Technologies. ESI mass spectra in positive mode were acquired with a Micromass Q-TOF I. High-resolution El mass spectra were acquired with a HRes, Concept S1, Magnetic Sector mass spectrometer and were conducted in the John L. Holmes Mass Spectrometry Facility at the Department of Chemistry and Biomolecular Sciences, University of Ottawa. Absorbance and emission spectra were recorded using a Cary 50 UV-Vis spectrophotometer and a PTI spectrofluorometer, respectively, using a quartz cuvette with a path length of $1 \mathrm{~cm}$. Laser flash photolysis was performed with a Q-switched Nd:YAG-laser (355 nm, $10 \mathrm{~mJ} / \mathrm{pulse})$ in a LFP-111 system (Luzchem Research Inc., Ottawa, Canada), in 1×1 $\mathrm{cm}$ LFP-Luzchem cuvettes or glass slides (Fisher Scientific). The absorbance of the samples at $355 \mathrm{~nm}$ was $\sim 0.3$.

\section{Single-Molecule Imaging and Analysis}

Single-molecule fluorescence experiments were performed within thin poly(methyl methacrylate) (PMMA) films containing the photochromefluorophore assembly 1a deposited over AgNP-functionalized glass coverslips. All glassware (vials, pipets and coverslips) for single-molecule experiments were cleaned with piranha solution $\left(\mathrm{H}_{2} \mathrm{O}_{2}: \mathrm{H}_{2} \mathrm{SO}_{4} 1: 3\right)$ for $1 \mathrm{~h}$ and then rinsed thoroughly with MilliQ water. The slide surface was functionalized with aminopropyltriethoxysilane (APTES) by immersing the coverslips in a $2 \% \mathrm{v} / \mathrm{v}$ aqueous APTES solution for $2 \mathrm{~h}$, then washing in an ultrasound bath with MilliQ water, and drying under a nitrogen flow. The nanoparticle monolayer was obtained by adding a drop of concentrated AgNP solution to the coverslip, rinsing after $1 \mathrm{~h}$ with water and then drying with nitrogen. Polymer films were spin coated $(2500 \mathrm{rpm}$, $45 \mathrm{sec}$.) from a $1 \% \mathrm{w} / \mathrm{w}$ PMMA solution in acetonitrile containing $1 \mathrm{a}$ or 2 (10-6 $\mathrm{mol} \mathrm{kg}^{-1}$ [pol]) onto the functionalized coverslips. Atomic Force Microscopy (AFM) imaging was performed under air using a Molecular Imaging PicoPlus AFM working in non-contact mode, using AFM probes from Budget Sensors (Tap150-G) with a nominal resonance frequency of $150 \mathrm{kHz}$ (force constant of $5 \mathrm{~N} \mathrm{~m}^{-1}$ ). Fluorescence imaging was performed with an Olympus FV1000 TIRF microscope (Olympus, Japan) equipped with He-Ne CW lasers (633 nm, 05-LHP-991 and 543 nm, 05LGP-193) and an EM-CCD (Rolera EM-C2, Q-Capture). The Olympus FV1000 is also coupled to a Fluorescence Lifetime Imaging (FLIM) system (MicroTime 200, PicoQuant, Germany). A beam splitter cube was used to reflect the excitation light into the oil immersion TIR (Total Internal Reflection) objective (100X, N.A. 1.45, Olympus, PLAPO). The fluorescence emission collected was in the spectral range of 655-725 nm. The laser power density at the sample was measured at $83 \mathrm{~W} / \mathrm{cm}^{2}$ at $100 \%$ of the laser power at $\lambda_{\mathrm{Ex}}=633 \mathrm{~nm}$, and $26 \mathrm{~W} \mathrm{~cm}^{-2}$ at $\lambda_{\mathrm{Ex}}=543 \mathrm{~nm}$. Each frame of the TIRFM image sequences recorded consists of a $501 \times$ 502 pixel (px), $80 \times 80 \mu \mathrm{m}$ image with a pixel size of $159 \mathrm{~nm}$. Fluorescence spectra of stochastic emission (bursting events) were recorded with the coupled FLIM system, equipped with a frequency 
doubled picosecond pulse diode laser $(637 \mathrm{~nm}, 100 \mathrm{ps}, 40 \mathrm{MHz}$, LDH-PFA-530L, PicoQuant). The laser beam was collimated and focused through a fiber-coupling unit. A beam splitter Z638rdc (Chroma) was used to reflect the excitation light into the oil immersion TIR objective. The epi-fluorescent signal was passed through a $560 \mathrm{~nm}$ long pass filter and collected by a Shemrock SR-163 spectrograph (Andor Technology, South Windsor, USA). Analysis of TIRFM image sequences (100 frames/image sequence, integration time per frame $=999 \mathrm{~ms}$ ) was carried out using a combination of ImageJ $(\mathrm{NIH})$, MATLAB (MathWorks) and OriginLab software. In brief, $3 \times 3$ px regions of interest (ROIs) were selected based on the automated identification of stochastic emission representing the formation of $\mathbf{1 b}$. After background subtraction was performed with ImageJ (rolling ball algorithm) bursting events were examined graphically. This was done by first using ImageJ to measure the mean fluorescence intensity inside each ROI for every frame in a 100 $s$ image sequence. The data were tabulated and imported into OriginLab, where frame numbers were converted to units of time. Mean intensity was then plotted as a function of time to generate unique intensity profiles for every ROI. Graphical residual analysis was then performed in order to identify fluorescence bursting events, which are characterized by a variance of the signal residual exceeding the positive limit of the variance exhibited by a distribution of residuals representing scattering by AgNP only (Figure S6; compare with S7, Supporting Information). The latter was obtained from TIRFM imaging of PMMA spin coated atop AgNP in the absence of $\mathbf{1 a}, \mathbf{1 b}$ or $\mathbf{2}$. Further details on this protocol are available in the Supporting Information.

\section{Acknowledgements}

We acknowledge financial support from Natural Sciences and Engineering Research Council of Canada, Canada Research Chairs and Canadian Foundation for Innovation. S. Impellizzeri acknowledges the award of a Banting Postdoctoral Fellowship. S. Simoncelli acknowledges a travel grant from UBA and a Ph.D. fellowship from CONICET as well as a DFAIT (Canada) fellowship from ELAP (Emerging Leaders in the Americas Program). G. K. Hodgson acknowledges the award of an Ontario Graduate Scholarship. P. F. Aramendia is a staff member of CONICET (Carrera del Investigador Científico, Consejo Nacional de Investigaciones Científicas y Técnicas, Argentina).

Keywords: surface plasmon $\cdot$ nanoparticles $\cdot$ photochromes $\bullet$ TIRFM

[1] W. L. Barnes, A. Dereux, T. W. Ebbesen, Nature 2003, 424, 824-830.

[2] aK. L. Kelly, E. Coronado, Z. L. L., G. C. Schatz, J. Phys Chem. B 2003, 107, 668-677; bE. Hao, G. C. Schatz, J. Chem. Phys. 2004, 120, 357-367; cK. Stamplecoskie, J. C. Scaiano, J. Am. Chem. Soc. 2010, 132, 1825-1827; dK. Stamplecoskie, J. C. Scaiano, Photochem. Photobiol. 2012, 88, 762-768.

[3] aP. Anger, P. Bharadwaj, L. Novotny, Phys. Rev. Lett. 2006 96; bP. K. Jain, M. A. El-Sayed, Chem. Phys. Lett. 2010, 487, 153-164.

[4] aH. Nishi, T. Asahi, S. Kobatake, J. Phys. Chem. C 2009, 113 17359-17366; bR. Klajn, P. J. Wesson, K. J. M. Bishop, B. A. Grzybowski, Angew. Chem. Int. Ed. 2009, 48, 7035-7039; cY. Tsuboi, R. Shimizu, T. Shoji, N. Kitamura, J. Am. Chem. Soc. 2009, 131, 12623-12627; dB. Wu, K. Ueno, Y. Yokota, K. Sun, H. Zeng, H. Misawa, J. Phys. Chem. Lett. 2012, 3, 1443-1447.
[5] J. C. Crano, R. J. Guglielmetti, Organic Photochromic and Thermochromic Compounds, Plenum Press, New York, 1999.

[6] J. R. Lakowikz, Principles of Flourscence Spectroscopy, Springer, New York, 2006.

[7] J. Cusido, S. Impellizzeri, F. M. Raymo, Nanoscale 2011, 3, 59-70.

[8] G. Baffou, R. Quidant, F. J. García de Abajo, ACS Nano 2010 , 4, 709-716.

[9] aE. Deniz, M. Tomasulo, J. Cusido, I. Yildiz, M. Petrella, M. L. Bossi, S. Sortino, F. M. Raymo, J. Phys. Chem. C 2012, 116, 6058-6068; bE. Deniz, S. Sortino, F. M. Raymo, J. Phys. Chem. Lett. 2010, 1, 3506-3509.

[10] The kinetic analysis of the isomerization is discussed in the Supporting Information.

[11] H. Nabika, M. Takase, F. Nagasawa, K. Murakoshi, J. Phys. Chem. Lett. 2010, 1, 2470-2487.

[12] aA. Sundaramurthy, P. J. Schuck, N. R. Conley, D. P. Fromm, G. S. Kino, W. E. Moerner, Nano Lett. 2006, 6, 355-360; bK. Ueno, S. Juodkazis, V. Mizeikis, K. Sasaki, H. Misawa, Adv. Mater. 2008, 20, 26-30; cR. Alvarez-Puebla, L. M. Liz-Marzán, F. J. García de Abajo, J. Phys. Chem. Lett. 2010, 1, 24282434; dX.-Y. Zhang, A. Hu, T. Zhang, W. Lei, X.-J. Xue, Y. Zhou, W. W. Duley, ACS Nano 2011, 5, 9082-9092.

[13] S. Simoncelli, M. J. Roberti, B. Araoz, M. L. Bossi, P. F. Aramendía, Journal of the American Chemical Society 2014, $136,6878-6880$.

[14] In the case of a $90 \mathrm{~nm}$ spherical nanostructure, the volume where the field is higher than half of the intensity at the NP surface is approximately a cilinder of $20 \mathrm{~nm}$ in height and 90 $\mathrm{nm}$ in diameter, located at each side of the NP in the direction defined by the incident photon polarization.

[15] A. O. Govorov, H. H. Richardson, Nano Today 2007, 2, 30-38.

[16] aW. Wenseleers, F. Stellacci, T. Meyer-Friedrichsen, T. Mangel, C. A. Bauer, S. J. K. Pond, S. R. Marder, J. W. Perry, J. Phys. Chem. B. 2002, 106, 6853-6863; bM.-Q. Zhu, G.-F. Zhang, C. Li, M. Aldred, E. Chang, R. A. Drezek, A. D. Q. Li, J. Am. Chem. Soc. 2011, 133, 365-372.

[17] aO. Neumann, A. D. Neumann, E. Silva, C. Ayala-Orozco, S. Tian, P. Nordlander, N. J. Halas, Nano Letters 2015, 15, 78807885; bC. Fasciani, C. J. B. Alejo, M. Grenier, J. C. NettoFerreira, J. C. Scaiano, Organic Letters 2011, 13, 204-207. 
FULL PAPER 